\title{
Modelo experimental de bandagem ajustável do tronco pulmonar para preparo rápido do ventrículo
}

\author{
Carlos A. DIAS*, Renato S. ASSAD*, Luiz F. CANEO*, Maria Cristina D. ABDUCH*, \\ Vera D. AIELLO*, Altamiro Ribeiro DIAS*, Miguel BARBERO-MARCIAL*
}

RBCCV 44205-519

\begin{abstract}
Dias C A, Assad R S, Caneo L F, Abduch M C D, Aiello V D, Dias A R, Barbero-Marcial M - Modelo experimental de bandagem ajustável do tronco pulmonar para preparo rápido do ventrículo. Rev Bras Cir Cardiovasc 2000; 15(4): 328-37.
\end{abstract}

RESUMO: Objetivo: Um dispositivo de bandagem do tronco pulmonar (TP) com ajuste percutâneo foi desenvolvido com o objetivo de induzir a hipertrofia rápida do ventrículo subpulmonar.

Material e Métodos: Consiste de um manguito de silicone insuflável percutaneamente, através de um botão de silicone auto-selante. Os corações de 7 cabritos jovens (peso médio: $8,7 \mathrm{~kg}$ ) foram submetidos à sobrecarga sistólica imposta pelo dispositivo e avaliados sob os aspectos hemodinâmicos, ecocardiográficos e morfológicos. Foram extraídas biópsias basais do miocárdio do ventrículo direito (VD) para análise microscópica (perímetro e área do miócito). O dispositivo foi implantado no TP. As pressões do VD, TP e aorta foram monitorizadas. Após convalescença, foi iniciado o protocolo de insuflação do dispositivo de bandagem do TP. Foi injetada água destilada no botão auto-selante (via percutânea), até que a pressão sistólica do VD atingisse $70 \%$ da pressão sistólica do VE. As avaliações ecocardiográfica e hemodinâmica foram realizadas a cada 24 horas. A sobrecarga sistólica do VD foi mantida por um período de 96 horas, com insuflações progressivas no limite máximo tolerado, a cada 24 horas. Após esse período, os animais foram sacrificados para análise morfológica dos corações. Outros 9 cabritos (peso médio: 7,7 kg) foram utilizados como grupo controle, para análise do peso do VD.

Resultados: Após 96 horas de sobrecarga sistólica do VD, foi observado um aumento do gradiente sistólico VD/TP de $10,1 \pm 4,3 \mathrm{mmHg}$ para $60,0 \pm 11,0 \mathrm{mmHg}$ e da pressão sistólica no VD de $22,4 \pm 4,1 \mathrm{mmHg}$ para $71,0 \pm 10,0 \mathrm{mmHg}(\mathrm{p}<0,0001)$. O ecocardiograma revelou aumento da espessura muscular do VD, de $4,4 \pm 0,5 \mathrm{~mm}$ para $7,3 \pm 1,7 \mathrm{~mm}(\mathrm{p}=0,001)$. Quando comparado ao grupo controle, houve um ganho de peso de $74 \%$ do VD submetido à sobrecarga sistólica $(p<0,0001)$. À microscopia óptica, foi observado aumento de $27 \%$ no perímetro e de $69 \%$ na área dos miócitos $(p=0,0001)$.

Conclusões: $O$ dispositivo proposto neste trabalho é efetivo e facilmente ajustável por via percutânea, possibilitando a hipertrofia do ventrículo subpulmonar em 96 horas de sobrecarga sistólica. A melhora na ajustabilidade da bandagem do TP poderá permitir melhores resultados da operação de Jatene em dois estágios, nos pacientes com transposição das grandes artérias.

DESCRITORES: Ventrículo cardíaco, cirurgia. Ventrículo cardíaco, cirurgia, métodos. Procedimentos cirúrgicos cardíacos, métodos. Transposição dos grandes vasos. Bandagem de tronco pulmonar, dispositivo. Artéria pulmonar, cirurgia. Procedimentos cirúrgicos reconstrutivos, métodos.

Trabalho realizado na Divisão de Experimentação do Instituto do Coração do Hospital das Clínicas da Faculdade de Medicina da Universidade de São Paulo. São Paulo, SP, Brasil.

Apresentado ao 27을 Congresso Nacional de Cirurgia Cardíaca. Rio de Janeiro, RJ, 23 a 25 de março, 2000.

*Do Instituto do Coração do Hospital das Clínicas da Faculdade de Medicina da Universidade de São Paulo

Endereço para correspondência: Dr. Renato Assad. Instituto do Coração HCFMUSP - Divisão de Cirurgia. Av. Dr. Eneas de Carvalho Aguiar, 44. São

Paulo, SP CEP: 05403-000. Tel.: (11) 3069-5248. e-mail: rsassad@cardiol.br 
Dias C A, Assad RS, Caneo LF, Abduch M C D, Aiello V D, Dias A R, Barbero-Marcial M - Modelo experimental de bandagem ajustável do tronco pulmonar para preparo rápido do ventrículo. Rev Bras Cir Cardiovasc 2000; 15(4): 328-37.

\section{INTRODUÇÃO}

A operação de Jatene representa 0 tratamento de escolha para a transposição dos grandes vasos (TGA) (1). No entanto, após o período neonatal, o ventrículo esquerdo (VE) encontra-se desadaptado para sustentar a circulação sistêmica. Nesse caso, a correção deve ser realizada em dois estágios $(2,3)$. No primeiro, realiza-se a bandagem do tronco pulmonar (TP) e uma fístula arterial sistêmico-pulmonar, para preparar o VE. No segundo, realiza-se o tratamento cirúrgico definitivo.

Entretanto, os resultados da bandagem do TP para o preparo ventricular rápido demonstraram uma elevada morbi-mortalidade (4). Provavelmente, estes resultados estão relacionados à falta de ajustabilidade e à técnica de bandagem do TP, que é realizada de forma empírica. O grau da bandagem do TP pode ser inadequado ou impreciso, ocasionando uma sobrecarga sistólica aguda importante ao VE.

Desenvolvemos um dispositivo desenhado para ajustar a bandagem por via percutânea e induzir a hipertrofia rápida do ventrículo pulmonar. As alterações provocadas pelo dispositivo foram avaliadas em ventrículo direito (VD) de cabritos jovens, através de estudos hemodinâmicos, morfológicos e ecocardiográficos, após 96 horas de aplicação ininterrupta de sobrecarga sistólica progressiva.

\section{MATERIAL E MÉTODOS}

\section{Anestesia}

Sete cabritos jovens com idade entre 30 e 60 dias e peso médio de $8,7 \pm 2,4 \mathrm{~kg}$ foram estudados. Outros 9 cabritos, de idade semelhante e peso médio de $7,7 \pm 1,2 \mathrm{~kg}$, foram utilizados como grupo controle para análise do VD em condições fisiológicas. Os animais permaneceram 24 horas em jejum antes da operação. A indução anestésica foi feita com quetamina (20 mg. $\left.\mathrm{kg}^{-1}\right)$. Em seguida, o acesso venoso foi obtido através da veia jugular. A antibioticoterapia profilática foi iniciada imediatamente antes da operação com cefazolina $\left(100 \mathrm{mg} \cdot \mathrm{kg}^{-1}\right.$ por dia) e gentamicina (2 mg. $\mathrm{kg}^{-1}$ por dia) por via intramuscular, a cada 12 horas e mantida até 0 sacrifício. $O$ animal foi então sedado com nembutal (5 a $10 \mathrm{mg} \cdot \mathrm{kg}^{-1}$, endovenoso) e entubado. A ventilação mecânica foi mantida com oxigênio a $100 \%$ e volume corrente de $15 \mathrm{ml} . \mathrm{kg}^{-1}$ (Harvard 708, South Natick, MA, E.U.A.). O eletrocardiograma foi monitorizado através do software ACQknowledge 3.01 (Biopac Systems, Inc., Goleta, CA, EUA). O animal foi posicionado em decúbito lateral direito e preparado para procedimento estéril.

\section{Dispositivo de Bandagem do Tronco Pulmonar}

O dispositivo de bandagem é constituído por três partes: anel de bandagem, tubo extensor e botão de insuflação (Figura 1). $\mathrm{O}$ anel de bandagem (Hazen Everett Co., New Jersey, EUA) é um manguito hidráulico em forma de $\mathrm{U}$, com diâmetro interno de $10 \mathrm{~mm}$ e largura de $5 \mathrm{~mm}$, cuja parede externa é constituída por silicone espesso $(1 \mathrm{~mm})$ e rígido, o que impede sua distensão. A superfície interna apresenta uma fina camada distensível de silicone, cuja expansão comprime a luz do vaso, conforme a quantidade de líquido injetada no botão de insuflação. Nas duas extremidades do manguito, existem orifícios que possibilitam a passagem de pontos para fechamento e fixação do anel no tronco pulmonar. $O$ tubo extensor comunica 0 anel de bandagem com o botão de insuflação, com a finalidade de conduzir o líquido injetado no botão para o manguito. Apresenta diâmetro interno de $2 \mathrm{~mm}$ e comprimento de $25 \mathrm{~cm}$, sendo também feito em silicone. $O$ botão de insuflação (Bard Access System, Utah, EUA) é um reservatório circular feito em silicone auto-selante, cuja base apresenta uma placa metálica. O reservatório apresenta uma saída, que é conectada ao tubo extensor. Este botão é implantado no subcutâneo, permitindo assim a insuflação ou desinsuflação do anel de bandagem por via percutânea.

\section{Procedimento}

Foi realizada toracotomia lateral esquerda (4ํㅡㄹ espaço intercostal). O pulmão foi afastado para expor a aorta descendente. Esta artéria foi puncionada com cateter 16 (Bard) para monitorização da pressão arterial (ACQknowledge 3.01, Biopac Systems, Inc., Goleta, CA, EUA) e de gases sangüíneos (Nova Biomedical - SP Ultra C, Waltham, MA, EUA). O cateter foi fixado com sutura em bolsa de polipropileno 5-0 e exteriorizado através da parede torácica. A seguir, o saco pericárdico foi amplamente aberto para exposição da via de saída do VD. Foram implantados mais dois cateteres de monitorização hemodinâmica, um no VD e outro, distalmente, no tronco pulmonar. Assim como na aorta torácica, os cateteres foram fixados com sutura em bolsa de polipropileno 5-0 e exteriorizados através da parede torácica. $O$ tronco pulmonar foi então dissecado e isolado, para implante do anel de bandagem. $O$ anel foi fixado na adventícia do TP com polipropileno 5-0, posicionado acima do plano da valva pulmonar. O tubo extensor do anel foi exteriorizado através do terceiro espaço intercostal e conectado ao botão de insuflação, alojado no subcutâneo da parede torácica. $O$ botão de insuflação foi testado por punção com agulha de insulina, sendo todo o ar do sistema aspirado. Os cateteres de pressão foram mantidos heparinizados. Após a drenagem do tórax, as costelas foram aproximadas e 
Dias C A, Assad R S, Caneo L F, Abduch M C D, Aiello V D, Dias A R, Barbero-Marcial M - Modelo experimental de bandagem ajustável do tronco pulmonar para preparo rápido do ventrículo. Rev Bras Cir Cardiovasc 2000; 15(4): 328-37.

os tecidos moles foram fechados por planos. Cerca de seis horas de pós-operatório imediato, o dreno de tórax era removido, após a constatação de drenagem mínima, ausência de fístula bronco-pleural e boa expansibilidade pulmonar.

\section{Protocolo}

Todos os cabritos receberam digital $(0,5 \mu \mathrm{g} . \mathrm{kg}$ ${ }^{1}$.dia ${ }^{-1}$, via intramuscular). Após período de convalescença de três a oito dias, iniciou-se o protocolo de insuflação do dispositivo de bandagem do TP, para indução do estresse sistólico progressivo do VD. Após a leitura das pressões basais, foi injetada água destilada no botão de insuflação (via percutânea), até que a pressão sistólica do VD atingisse $70 \%$ da pressão sistólica do VE, desde que não provocasse uma queda da pressão sistólica sistêmica superior a $10 \%$. Caso o animal apresentasse alteração do ritmo cardíaco, desconforto respiratório e/ou agitação após insuflação do dispositivo, o volume era imediatamente reduzido para um valor tolerável pelo animal e mantido assim por 24 horas. As medidas de pressão foram realizadas a cada 24 horas. A sobrecarga sistólica do VD foi mantida por um período de 96 horas, com insuflações progressivas no limite máximo tolerado, a cada 24 horas. Após esse período, os animais foram sacrificados para análise morfológica dos corações.

\section{Estudos Ecocardiográficos}

Todos os animais foram submetidos ao ecocardiograma (Ultramark 4, Advanced Technology Laboratories, Bothell, WA, E.U.A.) no pré-operatório para confirmação da menor espessura do VD em relação ao ventrículo esquerdo. Após o início de insuflação do dispositivo de bandagem, o ecocardiograma foi realizado a cada 24 horas, até o sacrifício dos animais. Foram observados os seguintes parâmetros: 1- Espessuras das paredes livres do VD, VE e septo; 2- Gradiente VD/TP; 3Presença de efusões nas cavidades pleuropericárdicas; 4- Função ventricular. Foram utilizados transdutores de 2,5 e $5 \mathrm{mHz}$, apropriados para o tamanho do animal. As medidas de espessura das paredes ventriculares e septal foram feitas através do corte paraesternal transverso, em modo $M$. O Doppler contínuo foi utilizado para avaliar o gradiente VD/TP provocado pelo dispositivo de bandagem.

\section{Estudos Morfológicos}

Foram colhidas amostras do subepicárdio da parede anterior da via de saída do VD durante a condição basal (antes do preparo do VD) e fixadas em formalina tamponada $10 \%$, para estudo sob microscopia óptica. Após 96 horas de sobrecarga sistólica do VD, os animais foram sacrificados para retirada do coração. Os animais do grupo controle foram também sacrificados pela mesma rotina do grupo submetido à sobrecarga sistólica do VD, para comparação dos pesos do septo e dos ventrículos.

Medidas de Peso - Após a remoção dos corações, as paredes ventriculares e do septo foram separadas de acordo com a técnica de FULTON et al. (5), descrita a seguir. As estruturas atriais foram ressecadas no plano das valvas atrioventriculares e desprezadas, bem como os grandes vasos junto às valvas semilunares. A seguir, foi realizada a secção da via de saída do VD rente ao septo, na sua porção basal. Procedeu-se então à separação da parede livre do VD, da base para o ápice, rente ao septo interventricular. Similarmente, a parede livre do VE foi separada do septo. Os pesos do septo e das paredes livres dos ventrículos foram tomados em balança de precisão, considerando até centésimos de grama. Estas medidas de peso foram indexadas ao peso corporal do animal $\left(\mathrm{g} \cdot \mathrm{kg}^{-1}\right)$, devido à variação no tamanho dos animais, para permitir comparação entre os animais, conforme sugestão de BISHOP \& $\operatorname{COLE}^{(6)}$.

Microscopia Óptica - Após a pesagem, os músculos cardíacos foram fixados em formalina tamponada $10 \%$, por um período de 24 horas. Foi realizado um corte transversal da massa ventricular direita, um centímetro abaixo da junção atrioventricular, para obter secções das paredes anterior e posterior do VD. Após processamento histológico de rotina, secções com espessura de $5 \mu$ foram coradas com hematoxilina e eosina. 0 perímetro e área dos miócitos, seccionados transversalmente no nível do núcleo, foram medidos através do sistema de análise de imagem (Quantimet-Leica, Leica Cambridge Ltd., Cambridge, Reino Unido), com aumento de 400x. Foram anotadas aleatoriamente de cada condição, as medidas de 140 miócitos do VD dos animais do grupo controle (quatro campos por animal) e de 105 miócitos dos ventrículos submetidos à sobrecarga sistólica (três campos por animal).

\section{Análise Estatística}

Os valores estão expressos como médias \pm desvio padrão. Utilizou-se o teste $t$ de Student pareado para comparar os dados basais das medidas hemodinâmicas, ecocardiográficas e de microscopia óptica com os dados do grupo submetido à sobrecarga sistólica do VD. A comparação entre os valores dos pesos do septo e dos ventrículos do grupo controle com os do grupo submetido à sobrecarga sistólica do VD foi feita com o teste de Student não pareado. A significância estatística foi estabelecida em $5 \%$. Todas as variáveis consideradas foram avaliadas com 
Dias C A, Assad RS, Caneo LF, Abduch M C D, Aiello V D, Dias A R, Barbero-Marcial M - Modelo experimental de bandagem ajustável do tronco pulmonar para preparo rápido do ventrículo. Rev Bras Cir Cardiovasc 2000; 15(4): 328-37.

TABELA 1

\begin{tabular}{lccccc}
\hline \multicolumn{7}{c}{ MEDIDAS HEMODINÂMICAS PRÉ E PÓS 96 HORAS DE SOBRECARGA SISTÓLICA DO VD } \\
\hline $\begin{array}{l}\text { 96 HORAS DE } \\
\begin{array}{l}\text { SOBRECARGA } \\
\text { SISTÓLICA } \\
\text { DO VD }\end{array}\end{array}$ & $\begin{array}{c}\text { FREQÜÊNCIA } \\
\text { CARDÍACA AO } \\
\text { (Bpm) }\end{array}$ & $\begin{array}{c}\text { PRESSÃO } \\
\text { SISTÓLICA } \\
\text { (mmHg) }\end{array}$ & $\begin{array}{c}\text { PRESSÃO } \\
\text { SISTÓLICA } \\
\text { VD (mmHg) }\end{array}$ & $\begin{array}{c}\text { GRADIENTE } \\
\text { VD/TP } \\
\text { (mmHg) }\end{array}$ & $\begin{array}{c}\text { RAZÃO DE } \\
\text { PRESSÕES } \\
\text { VD/VE (\%) }\end{array}$ \\
\hline PRÉ & $127,9 \pm 12,5$ & $79,4 \pm 6,1$ & $22,4 \pm 4,1$ & $10,1 \pm 4,3$ & $28,5 \pm 6,4$ \\
PÓS & $150,0 \pm 8,7$ & $68,6 \pm 3,6$ & $71,0 \pm 10,0$ & $60,0 \pm 11,0$ & $103,5 \pm 13,6$ \\
Valor de $p$ & 0,014 & 0,005 & 0,0001 & 0,0001 & 0,0001 \\
\hline
\end{tabular}

Valores $=$ Média \pm desvio padrão. $n=7 . V D=$ ventrículo direito. $B p m=$ batimentos por minuto. $A o=$ aorta. TP = tronco pulmonar. VE = ventrículo esquerdo

TABELA 2 ACHADOS ECOCARDIOGRÁFICOS PRÉ E PÓS 96 HORAS DE SOBRECARGA SISTÓLICA DO VD

\begin{tabular}{ccccc}
\hline $\begin{array}{c}\text { 96 HORAS DE } \\
\text { SOBRECARGA } \\
\text { SISTÓLICA } \\
\text { DO VD }\end{array}$ & $\begin{array}{c}\text { PAREDE } \\
\text { LIVRE VD } \\
(\mathbf{m m})\end{array}$ & $\begin{array}{c}\text { SEPTO } \\
\text { INTERVENTRICULAR } \\
(\mathbf{m m})\end{array}$ & $\begin{array}{c}\text { PAREDE } \\
\text { LIVRE VE } \\
(\mathbf{m m})\end{array}$ & $\begin{array}{c}\text { GRADIENTE } \\
\text { VD/TP } \\
(\mathbf{m m H g})\end{array}$ \\
\hline PRÉ & $4,4 \pm 0,5$ & $4,9 \pm 0,4$ & $5,4 \pm 0,5$ & $10,6 \pm 5,4$ \\
PÓs & $7,3 \pm 1,7$ & $5,1 \pm 0,4$ & $5,1 \pm 0,4$ & $74,9 \pm 9,7$ \\
Valor de p & 0,002 & NS & NS & 0,0001 \\
\hline
\end{tabular}

Valores $=$ Média \pm desvio padrão. $-n=7 . V D=$ ventrículo direito. $V E=$ ventrículo esquerdo. $T P=$ tronco pulmonar. $N S=$ não significativo

o teste de normalidade de Kolmogorov-Smirnov, isto é, tinham distribuição normal. O software utilizado para análise estatística foi GB-STAT (Dynamic Microsystems, Inc., MD, EUA).

\section{RESULTADOS}

Durante o protocolo, os animais permaneceram ativos, sem sinais clínicos de descompensação cardíaca direita. Todos os dispositivos implantados funcionaram adequadamente, tanto para injetar quanto para aspirar o líquido do manguito. A quantidade de líquido utilizada para insuflar o manguito variou de 0,1 a $0,4 \mathrm{ml}$. Não foram observadas migração ou rotura do dispositivo, nem lesão das estruturas adjacentes decorrente do uso do manguito ou vazamentos através da única conexão, entre o tubo extensor e o botão auto-selante de insuflação.

\section{Medidas Hemodinâmicas}

Todos os animais apresentavam-se mais taquicárdicos ao final do protocolo, apesar do uso de digitálico (basal: 127,9 $\pm 12,5$ bpm; pós 96 horas de sobrecarga sistólica: 150,0 $\pm 8,7$ bpm; $p=0,003$ ).
Foi observada queda de $13,6 \%$ da pressão sistólica da aorta ao longo do protocolo, embora a função do VE estivesse preservada, segundo avaliação ecocardiográfica ( $p=0,005)$.

No período de 96 horas de sobrecarga sistólica, o VD apresentou um aumento na pressão sistólica de $216,7 \%$, isto é, de $22,4 \pm 4,1 \mathrm{mmHg}$ para 71,0 $\pm 10,0 \mathrm{mmHg}$, superando a pressão sistólica do VE $(p=0,0001)$. $O$ gradiente sistólico entre $O V D$ e $o$ TP atingido durante o protocolo variou de 10,1 $\pm 4,3$ $\mathrm{mm} \mathrm{Hg}$ para $60,0 \pm 11,0 \mathrm{~mm} \mathrm{Hg}(p=0,0001)$. Paralelamente, a razão de pressões VD/VE passou de $28,5 \pm 6,4 \%$ antes da sobrecarga sistólica do VD para $103,5 \pm 13,6 \%$ após o período de 96 horas do protocolo $(p=0,0001)$. As medidas hemodinâmicas pré e pós 96 horas de sobrecarga sistólica do VD estão resumidas na Tabela 1.

\section{Achados Ecocardiográficos}

A Tabela 2 mostra os dados ecocardiográficos pré e pós 96 horas de sobrecarga sistólica do VD. Observamos que o período de 96 horas de sobrecarga sistólica do VD foi suficiente para aumentar em $66 \%$ a espessura da parede livre deste ventrículo (de $4,4 \pm 0,5 \mathrm{~mm}$ para $7,3 \pm 1,7 \mathrm{~mm} ; \mathrm{p}=0,002$ ), supe- 
Dias C A, Assad R S, Caneo L F, Abduch M C D, Aiello V D, Dias A R, Barbero-Marcial M - Modelo experimental de bandagem ajustável do tronco pulmonar para preparo rápido do ventrículo. Rev Bras Cir Cardiovasc 2000; 15(4): 328-37.

TABELA 3

MEDIDAS DE PESO DAS MASSAS MUSCULARES CARDÍACAS INDEXADAS AO PESO CORPORAL (g/kg)

\begin{tabular}{lccc}
\hline ANIMAIS & VD & SEPTO & VE \\
\hline Controle & $0,99 \pm 0,1$ & $1,24 \pm 0,22$ & $1,68 \pm 0,35$ \\
Treinados & $1,72 \pm 0,24$ & $1,32 \pm 0,20$ & $1,69 \pm 0,16$ \\
Valor de $p$ & 0,0001 & NS & NS \\
\hline
\end{tabular}

Valores $=$ Média \pm desvio padrão. Grupo controle: $n=9$. Grupo treinado (submetidos à 96 horas de sobrecarga sistólica do VD): $n=7$. $V D=$ ventrículo direito. $V E=$ ventrículo esquerdo. Septo $=$ septo interventricular. $N S=$ não significativo

TABELA 4

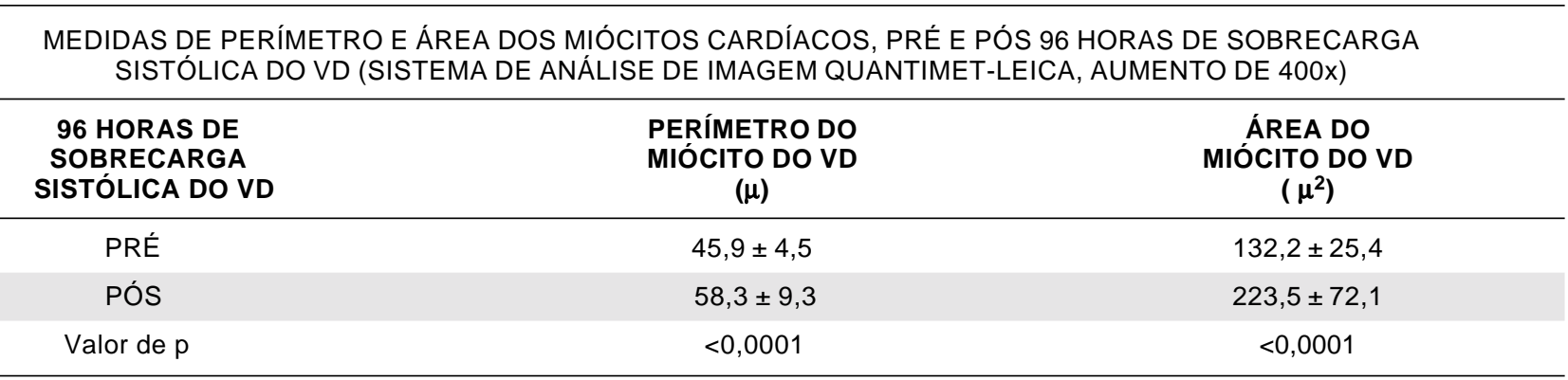

Valores $=$ Média \pm desvio padrão. $n=7 . V D=$ ventrículo direito

rando as medidas da parede livre do VE e do septo $(5,1 \pm 0,4 \mathrm{~mm})$. Não houve variação significativa das espessuras do septo interventricular e da parede livre do VE. O gradiente de pressão entre o VD e o TP, medido pelo Doppler, passou de 10,6 $\pm 5,4$ $\mathrm{mmHg}$ antes da sobrecarga sistólica do VD para $74,9 \pm 9,7 \mathrm{mmHg}$ após o período de 96 horas do protocolo $(p=0,0001)$. As medidas do gradiente de pressão VD/TP realizadas pelo Doppler, antes do período de sobrecarga sistólica do VD, "superestimaram" em 5\% as medidas hemodinâmicas lidas através dos cateteres implantados. As medidas do final do protocolo realizadas pelo Doppler foram "superestimadas" em $25 \%$ em relação à leitura de pressões através dos cateteres. A contratilidade do VE manteve-se preservada durante todo o protocolo, apesar de serem encontradas efusões pleurais (moderada quantidade) em todos os animais.

\section{Achados Morfológicos}

Peso dos Ventrículos - O peso do VD dos animais submetidos ao período de 96 horas de sobrecarga sistólica foi $74 \%$ maior que o do grupo controle $(0,99 \pm 0,17 \mathrm{~g} / \mathrm{kg}$ versus $1,72 \pm 0,24 \mathrm{~g} / \mathrm{kg} ; \mathrm{p}=0,0001)$. Não houve diferença de peso do septo interventricular e do VE entre os animais submetidos à sobrecarga sistólica do VD e o grupo controle. Estas medidas de peso estão resumidas na Tabela 3 .
Microscopia Óptica - A Tabela 4 mostra os resultados do perímetro e da área das fibras cardíacas, medidos antes e depois da sobrecarga sistólica do VD. Foi observado aumento significativo do perímetro do miócito do VD da ordem de $27 \%$, após 96 horas de sobrecarga sistólica (de 45,9 \pm 4,5 $\mu$ nas amostras basais, para 58,3 \pm 9,3 $\mu$ após 0 protocolo; $\mathrm{p}<0,0001)$. A Figura 2 apresenta fotos representativas dos miócitos cardíacos do animal \# 6 , antes e depois do protocolo de sobrecarga sistólica do VD. Foi claramente observado um aumento significativo de $69 \%$ da área dos miócitos cardíacos após o protocolo (de $132,2 \pm 25,4 \mu^{2}$ nas amostras basais, para 223,5 $\pm 72,1 \mu^{2}$ após o protocolo; $p<0,0001)$. Não foram observados fibrose intersticial ou edema importante.

\section{COMENTÁRIOS}

O dispositivo apresentado neste estudo representa uma opção interessante não apenas para o primeiro estágio do preparo rápido do VE, em pacientes com TGA e septo interventricular íntegro, como também na conversão da correção da TGA no plano atrial (Senning ou Mustard) para a operação de Jatene ${ }^{(7-9)}$. Ele foi desenhado para manipular de modo variável e reversível a pós carga do ventrículo subpulmonar, sem limite de tempo. Assim, um 
Dias C A, Assad R S, Caneo LF, Abduch M C D, Aiello V D, Dias A R, Barbero-Marcial M - Modelo experimental de bandagem ajustável do tronco pulmonar para preparo rápido do ventrículo. Rev Bras Cir Cardiovasc 2000; 15(4): 328-37.

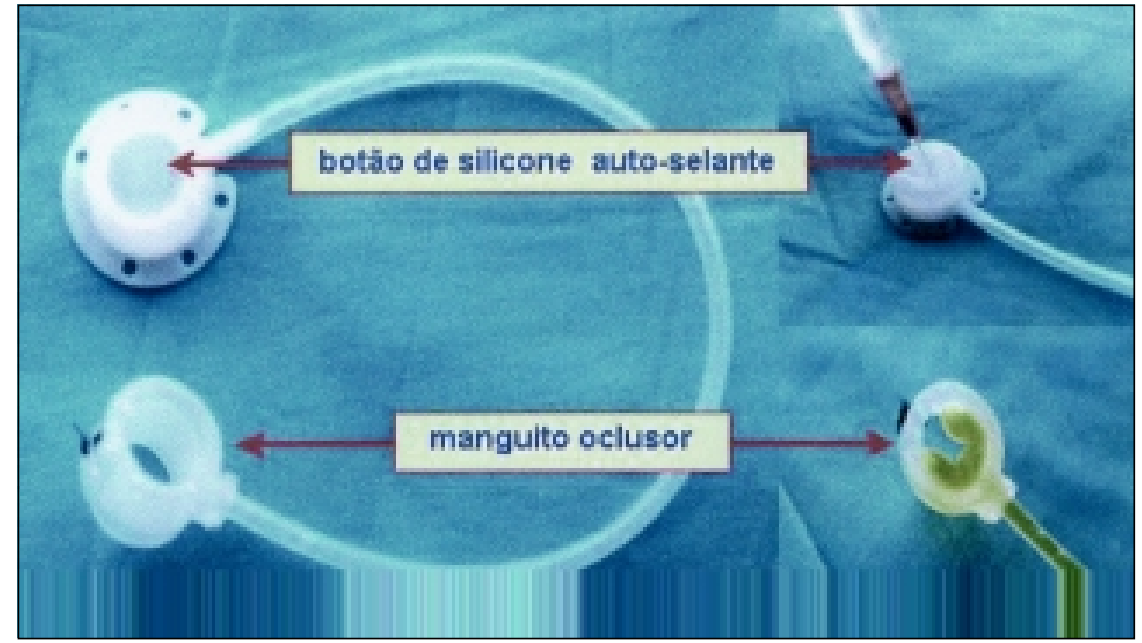

Fig. 1- Dispositivo de bandagem do tronco pulmonar, constituído por três partes: anel de bandagem, tubo extensor e botão de insuflação auto selante.

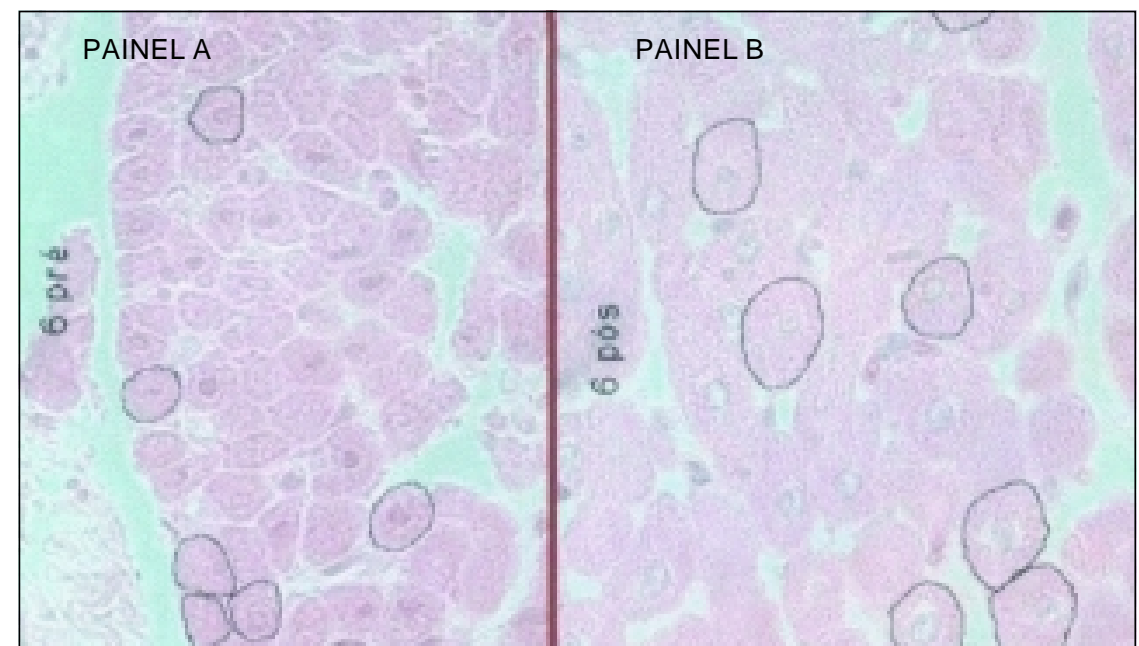

Fig. 2 - Foto de miócitos cardíacos do VD do animal \#6, seccionados transversalmente no nível do núcleo, indicando a medida do perímetro e sua respectiva área. (Painel A: amostras basais, Painel B: pós 96 horas de sobrecarga sistólica; aumento: 400 x).

estresse sistólico progressivo pode ser aplicado no ventrículo subpulmonar, limitando a gravidade da estenose imposta agudamente ao mesmo, durante a bandagem convencional.

\section{Crítica da Preparação}

Este protocolo analisa o comportamento da hipertrofia aguda do VD de cabritos jovens. Não se sabe se a resposta ao estímulo de sobrecarga sistólica variável no ventrículo subpulmonar de animais adultos seria a mesma, no caso da aplicação clínica da operação de Jatene para tratar ventrículo direito falido em pacientes submetidos previamente à operação de
Senning ou Mustard. Na experiência clínica de MAVROUDIS E BACKER ${ }^{(8)}$, o tempo médio entre a bandagem convencional e a conversão da correção no nível atrial para a operação de Jatene foi de 15,6 meses, em pacientes com idade variando entre 1,9 a 23 anos.

Não foi avaliada alteração da hematose decorrente da bandagem do tronco pulmonar. Entretanto, não foram observados sinais clínicos de hipóxia e/ ou acidose importantes. Embora um ajuste mais preciso do grau de sobrecarga sistólica possa ser atingido com este dispositivo, sem prejuízo do fluxo pulmonar, este modelo não reflete a realidade clínica dos pacientes portadores de TGA e septo interventricular funcionalmente íntegro. A bandagem 
Dias C A, Assad R S, Caneo L F, Abduch M C D, Aiello V D, Dias A R, Barbero-Marcial M - Modelo experimental de bandagem ajustável do tronco pulmonar para preparo rápido do ventrículo. Rev Bras Cir Cardiovasc 2000; 15(4): 328-37.

do TP pode não ser tolerada com a insuflação do dispositivo em pacientes com hipóxia importante, sem um "shunt" sistêmico-pulmonar. Por outro lado, o presente estudo promove a hipertrofia do ventrículo subpulmonar sem maiores alterações das pressões de enchimento do átrio e/ou ventrículo. Este fato poderia ajudar a manter um "shunt" interatrial equilibrado em pacientes portadores de TGA, evitandose assim a operação de Blalock-Taussig. Além do mais, o dispositivo poderia ser desinsuflado a qualquer momento, caso ocorresse hipóxia grave.

\section{Aspectos da Literatura}

Foram abordados nesta revisão apenas alguns aspectos históricos relacionados conceitualmente ao nosso protótipo.

A idéia de dispositivos de bandagem ajustável compostos de um manguito hidráulico e botão autoselante é bem antiga. Em 1957, JACOBSON \& MCALLISTER ${ }^{(10)}$ propuseram engenhoso dispositivo de concepção semelhante ao proposto neste estudo, com o objetivo de desenvolver insuficiência cardíaca experimentalmente. Este dispositivo consistia de um manguito de borracha, com uma abertura lateral conectada à um reservatório protegido com borracha auto-selante. Foi aplicado experimentalmente em grandes vasos de cães, porém houve complicações de manuseio do dispositivo decorrentes da solidificação do contraste no reservatório.

BISHOP \& COLE (6), em 1969, utilizaram o dispositivo de Jacobson modificado com revestimento de silicone na borracha do manguito, exclusivamente para produzir insuficiência cardíaca direita. Tal modificação poderia diminuir a reação tecidual local. Estes autores depararam com dois grandes problemas: 1- havia muitas conexões, que acarretavam vazamentos com freqüência; 2- o manguito apresentava distensão centrífuga quando insuflado, perdendo sua efetividade sobre o tronco pulmonar. Nestes modelos, a parede externa do manguito era tão distensível quanto à parede sua interna.

Em 1972, EDMUNDS et al. (11) propuseram dispositivo de bandagem com ajuste percutâneo de concepção semelhante à proposta de Jacobson et al. ${ }^{(10)}$, aprimorando porém o dispositivo através da utilização de novos materiais e introduzindo uma camada externa não distensível no manguito hidráulico. A borracha foi substituída pelo silicone. O dispositivo foi avaliado em cães por um período de até 32 meses. Complicações inerentes à insuflação assimétrica do dispositivo, rotura do manguito e vazamento do material injetado contra-indicavam o seu uso clínico. Apesar disto, este trabalho representa um marco para o desenvolvimento dos dispositivos subseqüentes.
Em 1985, PARK et al. (12) também propuseram dispositivo do tipo manguito hidráulico, ajustável por via extratorácica, pelo sistema de punção de material auto-selante. A principal diferença dos dispositivos anteriores foi a presença de uma válvula unidirecional entre o botão auto-selante e o tubo conector ao manguito, para reter o líquido injetado no manguito e evitar vazamentos através do botão. Este recurso apresentava o inconveniente da não reversibilidade, no caso da não tolerância do volume injetado. Esta válvula unidirecional faz-se desnecessária, caso o sistema auto-selante no botão de insuflacão seja eficiente. Neste mesmo ano, SOLIS et al. ${ }^{\left({ }^{3}\right)}$ propuseram dispositivo semelhante aos de JACOBSON \& MCALLISTER (10), BISHOP \& COLE ${ }^{(6)}$, EDMUNDS et al. ${ }^{(11)}$ e PARK et al. ${ }^{(12)}$, porém com reforço na parede externa do manguito, para evitar problemas de distensão centrífuga do manguito ocorrida em estudos prévios. Pela primeira vez na literatura, foi mencionada a intenção de utilizá-lo no preparo do ventrículo subpulmonar para a operação de Jatene. A hipertrofia do VD foi documentada experimentalmente após 30 dias, através do ecocardiograma. Apesar das modificações propostas no dispositivo, não foi possível atingir gradiente elevado através da bandagem, devido à deformação do manguito hidráulico ocasionada pelo regime de pressão elevada contra o dispositivo. Este fato não foi observado no presente estudo, pois uma diferença importante do nosso protótipo em relação aos anteriores reside no fato de apresentar um reforço deliberado na espessura do anel externo do manguito, o que impede sua distensão centrífuga quando insuflado.

\section{Aspectos Ecocardiográficos}

A estimativa do gradiente pressórico pelo Doppler é baseada no princípio físico da conservação da energia, de acordo com a aceleração do fluxo sangüíneo através da bandagem. O gradiente é medido através da equação simplificada de Bernoulli, ou seja, $\Delta \mathbf{P}=\mathbf{4} \mathbf{V}^{\mathbf{2}}$, onde $\Delta \mathrm{P}=$ gradiente pressórico e $V=$ velocidade do fluxo sangüíneo. Esta equação assume que toda a energia de pressão convertida em energia cinética é perdida como turbulência distal à bandagem. No nosso protocolo, as medidas do gradiente de pressão entre VD e TP, avaliadas pelo Doppler, "superestimaram" as medidas hemodinâmicas lidas através dos cateteres implantados. Estas aparentes diferenças entre tais medidas podem ser explicadas por dois motivos principais. Primeiro, o pico de gradiente medido pelo Doppler difere do gradiente pico a pico medido pelo cateter, isto é, o pico de velocidade obtido pelo Doppler corresponde à diferença máxima entre as pressões instantâneas do VD e TP em qualquer ponto durante o período de ejeção sistólica. Por outro lado, o gradiente pico a pico medido pelo cateter representa 
uma medida não simultânea das pressões de VD e TP. Pelo fato destes picos de pressão ocorrerem em diferentes fases no período de ejeção, esta medida de gradiente pico a pico torna-se uma medida não fisiológica. Em segundo lugar, uma explicação plausível para a "superestimativa" do gradiente pressórico da bandagem pode estar relacionada com o efeito de estenose tipo túnel (ou segmentar), não pontual (como ocorre nas estenoses valvares), provocado pelo tamanho do manguito de bandagem utilizado neste protocolo. Esta situação pode ser comparada ao efeito Venturi, que permite a recuperação quase completa da pressão pós bandagem, ou seja, a energia da pressão é quase totalmente convertida em energia cinética no ponto máximo do estreitamento da bandagem. Neste caso, a energia cinética não é completamente dissipada como turbulência, como ocorre nas estenoses pontuais, sendo que grande parte desta energia cinética é reconvertida em energia de pressão pós bandagem. Conseqüentemente, a pressão medida diretamente pelo cateter aumenta, diminuindo assim o gradiente pressórico obtido através dos cateteres ${ }^{(14)}$. Nossos dados são corroborados pelos estudos in vitro de LEVINE et al. ${ }^{(15)}$, os quais demonstraram que há uma maior recuperação da pressão pós estenose diretamente relacionada à extensão da obstrução, ou seja, quanto mais longo o segmento estenótico, maior será a pressão pós obstrução medida diretamente pelo cateter, o que diminui o gradiente pressórico. Estes dados sugerem uma importante vantagem para a avaliação do gradiente pressórico através do Doppler, o qual, pelo fato de utilizar a velocidade pico, fornece o gradiente máximo na "vena contracta" (região de maior estreitamento da passagem do fluxo sangüíneo), determinando de modo mais fidedigno a sobrecarga sistólica imposta na câmara proximal.

\section{Aspectos Morfológicos}

O importante aumento de peso do VD submetido à sobrecarga sistólica poderia refletir um aumento do conteúdo de água (edema) do miocárdio. $\mathrm{Na}$ análise microscópica, entretanto, não foram evidenciados edema nem fibrose intersticial importantes. Estudos de KATAYAMA et al. ${ }^{(16)}$ revelaram que $o$ aumento do peso dos ventrículos submetidos à sobrecarga sistólica foi acompanhado de um aumento do peso seco do miocárdio, atribuindo portanto a um fenômeno de hipertrofia do miócito cardíaco e não ao edema do miocárdio. Entretanto, a magnitude do ganho de massa muscular necessária para a operação de Jatene permanece obscura. Outros parâmetros utilizados por diversos autores para indicar a operação primária de Jatene incluem a pressão do ventrículo subpulmonar acima de $65 \mathrm{mmHg}^{(17)}$ ou razão de pressões sistólicas entre VE/VD acima de $75 \%{ }^{(18)}$.
Quanto à análise microscópica da hipertrofia do miócito cardíaco, as medidas de perímetro e da área da fibra em corte transverso ao nível do núcleo está baseada nos estudos de ANVERSA et al.(19). Estes autores analisaram miócitos cardíacos de ratos submetidos a 20 horas de estenose da aorta abdominal. Foi observado que, além do aumento da síntese protéica nos miócitos, houve um aumento de $20 \%$ na área dos mesmos. Durante um período mais prolongado (13 dias) de sobrecarga sistólica similar, estes autores encontraram um aumento superior a $200 \%$ na área transversal dos miócitos cardíacos de ratos ${ }^{(20)}$. Estudos prévios em nosso laboratório, utilizando porém um cateter balão para induzir a hipertrofia aguda do VD de cabritos, durante um período variável de 9 a 20 dias, revelaram um aumento de $20 \%$ no diâmetro (não na área) dos miócitos cardíacos ${ }^{(21,22)}$. O presente estudo de 96 horas de sobrecarga sistólica induziu a um aumento de $69 \%$ na área dos miócitos cardíacos, o que foi suficiente para gerar pressões sistêmicas no ventrículo direito dos cabritos. $\mathrm{Na}$ realidade, ainda não há consenso quanto ao tempo nem a forma ideais de preparo do ventrículo subpulmonar para a operação de Jatene em dois estágios. Existem evidências de que a bandagem não gradual, ocasionando sobrecarga sistólica súbita e aguda, pode induzir a dano miocárdico importante ${ }^{(23)}$. Um programa de "recondicionamento físico" para o ventrículo subpulmonar deve ser estabelecido, objetivando uma hipertrofia a mais fisiológica possível.

\section{Implicações}

Foram utilizados como critérios para a insuflação do manguito a elevação da pressão sistólica do VD a $70 \%$ da pressão sistólica do VE e/ou uma queda máxima da pressão sistólica sistêmica de 10\%. Outros autores utilizam critérios imprecisos, tais como bem estar do animal ou sinais de descompensação do VD. SOLIS et al. (24) utilizaram, como critério, o máximo de constrição tolerável pelo animal, parâmetro de caráter subjetivo.

O tempo de sobrecarga sistólica de 96 horas foi eleito, mediante estudos prévios de hipertrofia do ventrículo subpulmonar de cabritos jovens, obtida durante 6 dias de bandagem do TP com cateter balão, porém com um regime mais gradual $(19,20)$. Interessante observar que, no presente estudo, alguns animais foram capazes de desenvolver pressão sistêmica no ventrículo subpulmonar com apenas 48 horas de sobrecarga sistólica. Entretanto, BOUTIN el al. ${ }^{(25)}$ associa a disfunção ventricular esquerda tardia a uma sobrecarga aguda extrema em pacientes submetidos à operação de Jatene após o preparo rápido do VE. Esta disfunção foi 
Dias C A, Assad R S, Caneo L F, Abduch M C D, Aiello V D, Dias A R, Barbero-Marcial M - Modelo experimental de bandagem ajustável do tronco pulmonar para preparo rápido do ventrículo. Rev Bras Cir Cardiovasc 2000; 15(4): 328-37.

inversamente proporcional a uma hipertrofia mais rápida e a uma disfunção mais grave após a bandagem do TP.

Talvez o nosso modelo de sobrecarga sistólica variável e progressiva possa favorecer uma hipertrofia do ventrículo subpulmonar mais saudável, quando comparado à bandagem convencional fixa, onde o estresse sistólico é abrupto e constante. O aumento gradual da pós carga do VD permitiu não apenas a sua tolerância progressiva à estenose do TP produzida pelo dispositivo, como também sua capacidade de gerar pressões até mesmo superiores à do VE após o período de treinamento.

Futuros estudos, utilizando a biologia molecular como ferramenta para analisar marcadores de hipertrofia patológica, poderão comparar diversas formas de treinamento do ventrículo subpulmonar. O objetivo final seria o de minimizar os danos celulares, maximizar a eficiência da bandagem pul- monar, elucidando assim a melhor forma de treinamento do ventrículo subpulmonar no preparo para a operação de Jatene.

\section{CONCLUSÃO}

O dispositivo proposto neste trabalho é biocompatível e de fácil implante. Foi eficiente em realizar a bandagem ajustável do tronco pulmonar por via percutânea. A sobrecarga sistólica imposta pelo dispositivo possibilitou a hipertrofia rápida (96 horas) do ventrículo pulmonar dos animais estudados. Esta abordagem poderá permitir o preparo do ventrículo subpulmonar não apenas para o primeiro estágio do preparo rápido do VE em pacientes com TGA e septo interventricular íntegro, como também na conversão do Senning ou Mustard falidos para a operação de Jatene.

RBCCV 44205-519

Dias C A, Assad R S, Caneo L F, Abduch M C D, Aiello V D, Dias A R, Barbero-Marcial M - Experimental model of adjustable pulmonary trunk banding for rapid prepare of the ventricle. Rev Bras Cir Cardiovasc 2000; 15(4): 328-37.

ABSTRACT: Objective: A device for pulmonary trunk (PT) banding with percutaneous adjustment was developed with the aim of inducing rapid hypertrophy of the subpulmonary ventricle.

Material and Methods: It consists of a silicon cuff occluder which holds the pulmonary trunk; an extension tube that connects the cuff to an auto-sealing silicon button, in which water is injected percutaneously to inflate the cuff and adjust the systolic load to the subpulmonary ventricle. The hearts of seven young goats (average wt.: $8.7 \mathrm{~kg}$ ) were submitted to systolic overload imposed by the device and assessed under hemodynamic, echocardiographic and morphologic aspects. Baseline myocardial samples of the right ventricular outflow tract were harvested for microscopic analysis (perimeter and area of the myocyte). The device was then placed on the PT. The pressures in the right ventricle (RV), PT and aorta were monitored. After convalescence, the protocol of insufflation of the banding device was initiated in order to achieve a RV systolic pressure $70 \%$ of the left ventricle systolic pressure. The hemodynamic and echocardiographic evaluations were performed every 24 hours. The RV systolic overload was maintained through a 96 hours period, with gradual insufflations of the cuff every 24 hours, according to the tolerance of the animal to the pressure load. After this period, the animals were killed for morphologic evaluation of the heart. Another nine goats (average wt.: $7.7 \mathrm{~kg}$ ) were used as control group to compare the ventricle muscle weight.

Results: After 96 hours of RV systolic overload, it was observed an increase in systolic gradient RV/PT, from $10.1 \pm 4.3 \mathrm{mmHg}$ to $60,0 \pm 11,0 \mathrm{mmHg}$ and in the RV systolic pressure, from $22.4 \pm 4.1 \mathrm{mmHg}$ to $71.0 \pm 10.0$ $\mathrm{mmHg}(\mathrm{p}<0.0001)$. Serial echocardiography showed an increase of the RV thickness, from $4.4 \pm 0.5 \mathrm{~mm}$ to $7.3 \pm 1.7 \mathrm{~mm}(p=0.001)$. Regarding RV weight, there was a $74 \%$ increase in the RV submitted to the systolic overload, when compared to the control group $(p<0.0001)$. Under optic microscopy, a $27 \%$ increase in perimeter and $69 \%$ increase in area of the myocytes were observed $(p=0.0001)$.

Conclusions: The device considered in this work is effective and easily adjustable percutaneously, suggesting that the prepare of the subpulmonary ventricle could probably be accomplished in a 96 hour period of systolic overload. Improvements in the adjustment of the PT banding may allow better results of the twostage Jatene operation in patients with transposition of the great arteries.

DESCRIPTORS: Heart ventricle, surgery. Heart ventricle, surgery, methods. Cardiac surgical procedures, methods. Transposition of great arteries. Pulmonary trunk banding device. Pulmonary artery, surgery. Reconstructive surgical procedures, methods. 
Dias C A, Assad R S, Caneo LF, Abduch M C D, Aiello V D, Dias A R, Barbero-Marcial M - Modelo experimental de bandagem ajustável do tronco pulmonar para preparo rápido do ventrículo. Rev Bras Cir Cardiovasc 2000; 15(4): 328-37.

AGRADECIMENTO: Agradecemos ao Sr. Nelson Correa Júnior, técnico de Laboratório, pela valiosa colaboração durante o protocolo.

\section{REFERÊNCIAS BIBLIOGRÁFICAS}

1 Jatene A D, Fontes V F, Paulista, P P et al. - Successful anatomic correction of transposition of the great vessels: a preliminary report. Arq Bras Cardiol 1975; 28: 461-4.

2 Yacoub M H, Radley-Smith R, MacLaurin R - Two-stage operation for anatomical correction of transposition of the great arteries with intact interventricular septum. Lancet 1977; 1: 1275-8.

3 Jonas R A, Giglia T M, Sanders S et al. - Rapid, twostage arterial switch for transposition of great arteries and intact ventricular septum beyond the neonatal period. Circulation 1989; 80 (suppl I):I 203-8.

4 Bosísio I B J - Avaliação do desempenho do ventrículo esquerdo na operação de Jatene em transposição das grandes artérias com septo ventricular íntegro após preparo rápido. [Tese. Doutorado] São Paulo: Faculdade de Medicina da Universidade de São Paulo, 1997.

5 Fulton R M, Hutchinson E C, Jones A M - Ventricular weight in cardiac hypertrophy. Br Heart $J$ 1952; 14 : 413-20.

6 Bishop S P \& Cole C R - Production of externally controlled progressive pulmonic stenosis in the dog. J Appl Physiol 1969; 26: 659-63.

7 Mee R B - Severe right ventricular failure after Mustard or Senning operation. Two-stage repair: pulmonary artery banding and switch. J Thorac Cardiovasc Surg 1986; 92: 385-90.

8 Mavroudis C \& Backer C L - Arterial switch after failed atrial baffle procedures for transposition of the great arteries. Ann Thorac Surg 2000; 69: 851-7.

9 Van Son J A, Reddy V M, Silverman N H, Hanley F L - Regression of tricuspid regurgitation after two-stage arterial switch operation for failing systemic ventricle after atrial inversion operation. J Thorac Cardiovasc Surg 1996; 111: 342-7.

10 Jacobson $\mathrm{J} \mathrm{H}$ \& McAllister F F - A method for the controlled occlusion of a larger blood vessels. Ann Surg 1957; 145: 334-43.

11 Edmunds L H Jr, Rudy L W, Heymann M A, Boucher J $\mathrm{K}$ - An adjustable pulmonary arterial band. Trans $A m$ Soc Artif Intern Organs 1972; 18: 217-25.

12 Park S C, Griffith B P, Siewers R D et al. - A percutaneously adjustable device for banding of the pulmonary trunk. Int J Cardiol 1985; 9: 477-84.
13 Solis E, Heck C F, Seward J B, Kaye M P Percutaneously adjustable pulmonary artery band. Ann Thorac Surg 1986; 41: 65-9.

14 Weyman A E - Clinical applications. In: Weyman A E, ed. Principles and practice of echocardiography. Phiiladelphia, Lea \& Febiger, 1994. p. 516-70.

15 Levine R A, Jimoh A, Cape E G, McMillan S, Yoganathan A P, Weyman A E - Pressure recovery distal to a stenosis: potential cause of gradient "overestimation" by Doppler echocardiography. J Am Coll Cardiol 1989; 13: $706-15$

16 Katayama $\mathrm{H}$, Krzeski R, Frantz E G et al. - Induction of right ventricular hypertrophy with obstructing balloon catheter: nonsurgical ventricular preparation for the arterial switch operation in simple transposition. Circulation 1993; 88: 1765-9.

17 Yasui $\mathrm{H}$, Kado H, Yonenaga K, Hisahara M et al. - Arterial switch operation for transposition of the great arteries, with special reference to left ventricular function. $J$ Thorac Cardiovasc Surg 1989; 98: 601-10.

18 Jonas R A - Update on the rapid two-stage arterial switch procedure. Cardiol Young 1991; 1: 99-100.

19 Anversa P, Vitali-Mazza L, Gandolfi A, Loud A V Morphometry and autoradiography of early hypertrophic changes in the ventricular myocardium of adult rat: a light microscopic study. Lab Invest 1975; 33: 125-9.

20 Anversa P, Hagopian M, Loud A V - Quantitative radioautographic localization of protein synthesis in experimental cardiac hypertrophy. Lab Invest 1973; 29: $282-92$

21 Assad R S, Cardarelli M, Abduch M C et al. - Bandagem reversível do tronco pulmonar: modelo experimental para o preparo rápido do ventrículo pulmonar. Rev Soc Bras Cir Cardiovasc, 1998; 13:239-48.

22 Assad R S, Cardarelli M, Abduch M C et al. - Reversible pulmonary trunk banding with a balloon catheter: assessment of rapid pulmonary ventricular hypertrophy. J Thorac Cardiovasc Surg 2000; 120: 66-72.

23 Redington A - Alternatives for LV training. CTSNet Discussion Forum, The Annals of Thoracic Surgery 2000. Arterial switch after failed atrial baffle procedures for transposition of the great arteries. http:/ /www.sts.org/section/atsdiscussion.

24 Solis E, Bell D, Alboliras H, Seward J, Kaye M P - Left ventricular preparation with an extrathoracically adjustable balloon occluder. Ann Thorac Surg 1987; 44: $58-61$

25 Boutin C, Wernovsky G, Sanders S P, Jonas R A, Castaneda A R, Colan S D - Rapid two-stage arterial switch operation: evaluation of left ventricular systolic mechanics late after an acute pressure overload stimulus in infancy. Circulation 1994; 90: 1294-303. 